\title{
Low penetrance in facioscapulohumeral muscular dystrophy type 1 with large pathological D4Z4 alleles: a cross-sectional multicenter study
}

Emmanuelle Salort-Campana ${ }^{1,2,3^{*}}$, Karine Nguyen ${ }^{2,3}$, Rafaelle Bernard ${ }^{2,3}$, Elisabeth Jouve ${ }^{4}$, Guilhem Solé5, Aleksandra Nadaj-Pakleza ${ }^{6}$, Julien Niederhauser ${ }^{7}$, Estelle Charles ${ }^{4}$, Elisabeth Ollagnon ${ }^{8}$, Françoise Bouhour ${ }^{9}$, Sabrina Sacconi ${ }^{10}$, Andoni Echaniz-Laguna ${ }^{11}$, Claude Desnuelle ${ }^{10}$, Christine Tranchant ${ }^{11}$, Christophe Vial ${ }^{9}$, Frederique Magdinier ${ }^{2}$, Marc Bartoli ${ }^{2}$, Marie-Christine Arne-Bes ${ }^{12}$, Xavier Ferrer ${ }^{5}$, Thierry Kuntzer ${ }^{7}$, Nicolas Levy ${ }^{2,3}$, Jean Pouget ${ }^{1,2}$ and Shahram Attarian ${ }^{1}$

\begin{abstract}
Background: Facioscapulohumeral muscular dystrophy type 1(FSHD1) is an autosomal dominant disorder associated with the contraction of D4Z4 less than 11 repeat units (RUs) on chromosome 4q35. Penetrance in the range of the largest alleles is poorly known. Our objective was to study the penetrance of FSHD1 in patients carrying alleles ranging between 6 to10 RUs and to evaluate the influence of sex, age, and several environmental factors on clinical expression of the disease.

Methods: A cross-sectional multicenter study was conducted in six French and one Swiss neuromuscular centers. 65 FSHD1 affected patients carrying a 4qA allele of 6-10 RUs were identified as index cases (IC) and their 119 at-risk relatives were included. The age of onset was recorded for IC only. Medical history, neurological examination and manual muscle testing were performed for each subject. Genetic testing determined the allele size (number of RUs) and the $4 \mathrm{qA} / 4 \mathrm{qB}$ allelic variant. The clinical status of relatives was established blindly to their genetic testing results. The main outcome was the penetrance defined as the ratio between the number of clinically affected carriers and the total number of carriers.
\end{abstract}

Results: Among the relatives, 59 carried the D4Z4 contraction. At the clinical level, 34 relatives carriers were clinically affected and 25 unaffected. Therefore, the calculated penetrance was $57 \%$ in the range of 6-10 RUs. Penetrance was estimated at $62 \%$ in the range of 6-8 RUs, and at $47 \%$ in the range of 9-10 RUs. Moreover, penetrance was lower in women than men. There was no effect of drugs, anesthesia, surgery or traumatisms on the penetrance.

Conclusions: Penetrance of FSHD1 is low for largest alleles in the range of 9-10 RUs, and lower in women than men. This is of crucial importance for genetic counseling and clinical management of patients and families.

Keywords: Facioscapulohumeral muscular dystrophy, Penetrance, FSHD1, D4Z4

\footnotetext{
* Correspondence: emmanuelle.salort-campana@ap-hm.fr

'AP-HM, Reference Center of Neuromuscular Disorders and ALS, Timone University Hospital, Aix-Marseille University, 264 rue Saint-Pierre, Marseille, Cedex 05 13385, France

${ }^{2}$ Aix Marseille Université - Inserm UMR_S 910 Medical Genetics and Functional

Genomics, Marseille, France

Full list of author information is available at the end of the article
}

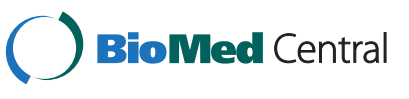

(c) 2015 Salort-Campana et al.; licensee BioMed Central. This is an Open Access article distributed under the terms of the Creative Commons Attribution License (http://creativecommons.org/licenses/by/4.0), which permits unrestricted use, distribution, and reproduction in any medium, provided the original work is properly credited. The Creative Commons Public Domain Dedication waiver (http://creativecommons.org/publicdomain/zero/1.0/) applies to the data made available in this article, unless otherwise stated. 


\section{Background}

Facioscapulohumeral muscular dystrophy (FSHD) is an autosomal dominant disorder with variable severity and inter- and intra-familial heterogeneity [1,2]. FSHD is characterized by a highly selective and asymmetrical pattern of muscle involvement. For two decades, the molecular diagnosis of FSHD type 1 (FSHD1) has been supported by the evidence of a heterozygous contraction of the D4Z4 repeat array from 1 to 10 repeat units (RUs) on $4 \mathrm{q} 35$ [3].

D4Z4 contraction is considered to be pathogenic if it occurs on a specific chromosomal background, i.e., (i) the presence of the 4A (159/161/168) haplotype and (ii) a single nucleotide polymorphism that creates a polyadenylation site (PAS) for the distal DUX4 transcript [4-6]. The unique association of FSHD1 with a specific haplotype remains controversial as some FSHD1 patients carry a D4Z4 contraction without the common 4A161PAS haplotype [7]. The pathological cut-off is conventionally determined at 10 RUs. Most FSHD1 patients carry 1 to 8 RUs on one allele. Large studies provided data on expressivity and penetrance in this range $[8,9]$. However, few studies reported the phenotypic spectrum associated with the upper pathological alleles (9-10 RUs). It was suggested that these patients present with a later and milder phenotype and a lower penetrance [10-14]. A better knowledge of penetrance in this range is necessary for improving genetic counseling, particularly in the context of predictive testing and prenatal diagnosis in FSHD families.

In our study, we aimed to estimate the penetrance in a large group of FSHD1 patients with alleles of 6-10 RUs. In addition, we evaluated the influence of sex, age, and several environmental factors on clinical expression of the disease.

\section{Methods}

A cross-sectional multicenter study was conducted in six French and one Swiss neuromuscular centers. Among the FSHD cases followed in the reference centers from 2007 to 2009, patients carrying a contracted D4Z4 array with an estimated size of $6 \mathrm{RUs}(27 \mathrm{~kb})$ to $10 \mathrm{RUs}$ $(40 \mathrm{~kb})$ and fulfilling the diagnostic criteria defined by the European Expert Group on FSHD [15] were selected to constitute the index case (IC) group. The second group included volunteer at-risk relatives with various degrees of kinship. For ethical considerations, we did not include patients less than 18 years of age or pregnant women.

At each center, patients and relatives were examined by a pluridisciplinary team that included a neurologist with neuromuscular disorder expertise, a geneticist and a physiotherapist. All clinical data were collected and blood was sampled for genetic analysis during a single visit.

All subjects enrolled in the study gave informed consent to participate. This study received the approval of the local ethic committee (Comité de Protection des Personnes Sud Mediterranée I).

\section{Clinical and functional evaluation}

For each IC or relative, we recorded the following: medical history, self-evaluation of physical activity, pedigree, clinical examination, functional assessment, and manual muscular test results. ICs and relatives were asked for information pertaining to physical activity (self evaluation of physical activity), number of trauma requiring an immobilization of one week or more, number of exposures to and types of anesthesia received, exposure to tobacco (number of pack-years of cigarettes), alcohol intake (average number of alcohol units per day and per week), and the use of any drugs. The first symptom experienced and the age of onset were recorded for ICs only. We did not record the age of onset in relatives because some of them had never been examined before and did not want their clinical status to be revealed. Therefore, we established the age at examination as the age of onset for clinically affected relatives. Neurologists examined the presence or absence of scapular winging, facial, limbs and thoraco-abdominal muscle weakness, selective involvement and asymmetry of muscle weakness. At the end of clinical examination, the neurologist blinded to the genetic results determined if the relative was clinically affected or not. The guidelines were to consider that a relative was clinically affected if there was facial and/or scapular fixator weakness; and there was absence of atypical signs suggesting an alternative diagnosis (including extraocular, masticatory, pharyngeal, or lingual muscle weakness and cardiomyopathy) $[10,15]$.

\section{Genetic testing}

Informed consent was obtained for every enrolled subject. In the relatives group, each participant was asked if he wanted to be informed about his own genetic results. Subjects who wished to be informed were given their genetic result by the geneticist during a genetic counseling session at the end of the study. The genetic result was validated by a second analysis on a new DNA sample. Each patient's blood sample was coded and anonymized.

All genetic analyses were performed at the Laboratory of Molecular Genetics in the Department of Medical Genetics in Marseille. DNA was prepared from isolated lymphocytes according to standard procedures. Restriction endonuclease digestion was performed and digested DNA was separated by pulsed-field gel electrophoresis (PFGE) in a $1 \%$ agarose gel. Allele sizes were estimated by Southern blot hybridization with a radiolabeled p13E-11 probe using $7 \mu \mathrm{g}$ of $E c o R I$ and $E c o R I / B \ln \mathrm{I}$ digested genomic DNA. In our laboratory practice and according to interlaboratory quality assessment, we consider that the pathological cut-off at 10 RUs corresponds to an EcoRI 
fragment of $40 \mathrm{~kb}$. Thus, the range of 6-10 RUs corresponds to fragments from $27-40 \mathrm{~kb}$. The molecular results in our diagnostic laboratory are provided in RUs. The $4 \mathrm{qA} / 4 \mathrm{qB}$ allelic variants were identified using $7 \mu \mathrm{g}$ of $\mathrm{Hin}$ dIII digested DNA, PFGE electrophoresis, and Southern blot hybridization with radiolabeled probes for $\mathrm{qA} / \mathrm{qB}$ according to standard procedures. The IC group was analyzed with EcoRI/p13E-11, EcoRI/BlnI/p13E-11, and HindIII/qAqB while the relatives group was analyzed by comparing their EcoRI/BlnI/p13E-11 pattern with that of the IC in each family. We considered as "carriers" the relatives harboring the contracted allele previously identified in the IC. We did not perform the analysis of the SSLPPAS sequence, as the recent literature indicates that only $50 \%$ of FSHD 1 patients carry the 4A161PAS permissive haplotype associated with the D4Z4 contraction. Thus, we did not consider this parameter as mandatory for molecular diagnosis [16].

\section{Statistical analyses}

To examine group comparability, we compared the age and sex of ICs, clinically affected carriers, clinically unaffected carriers, and non carriers. To study the influence of age, sex, and environmental factors on the risk of being clinically affected, we compared clinically affected versus clinically unaffected carriers.

For all mean comparisons, the Student's t-test or analysis of variance were used. Frequencies were compared using a chi-square test. When necessary, nonparametric tests (Mann-Whitney or Kruskal-Wallis tests) or Fisher exact tests were used. Differences were considered significant for all analyses when the $\mathrm{P}$ values were lower than 0.05 .

The risk of developing symptoms of the disease considering age, sex and number of RUs was estimated for clinically affected patients (IC and clinically affected carriers) versus clinically unaffected carriers using the multivariate logistic regression model. Odds ratios (ORs) and $95 \%$ confidence intervals (CIs) were calculated for age (continuous variable), sex and number of RUs classified into two categories (6-8 RUs, 9-10 RUs).

To estimate the penetrance, the primary criterion was the clinical status established by neurologists blinded with regard to genetic status. Penetrance was defined as the ratio between the number of carriers considered as clinically affected and the total number of carriers. In order not to underestimate the penetrance by taking in account the at-risk relatives only, we estimated the penetrance as a range between two figures including or excluding the ICs.

The Kaplan-Meier survival analysis was used for ICs and clinically unaffected carriers to estimate the agespecific cumulative first symptom incidence with the corresponding $95 \%$ CIs. For each IC, the time from birth to the earliest declarative age at onset of symptoms was collected. For clinically unaffected carriers, we considered the age at the time of the clinical evaluation.

\section{Results}

184 subjects were included in the study, 65 ICs and 119 relatives (Figure 1). Forty-four families encompassed less than 4 individuals and 21 families encompassed between 4 and 9 individuals. All ICs harbored a 4qA contracted allele. Among the 119 relatives, 59 were carriers of the D4Z4 contraction identified in the IC, and 60 were non carriers. Among the 59 relatives, 34 were clinically affected carriers and 25 were unaffected carriers.

Overall, 124 were carriers of the contraction, of which 99 individuals were clinically affected (65 ICs and 34 affected relatives).

\section{Demographic characteristics of the subjects}

Descriptive statistics of age and sex are described in Table 1 . Of the 99 clinically affected individuals (ICs and affected carriers), there were 62 men and 37 women. In the remaining unaffected carrier patients group, there were significantly more women (18 of $25, \mathrm{p}=0.002$ ). The age of the patients at the time of clinical evaluation did not significantly differ between ICs, affected carriers, unaffected carriers, and non carriers. Table 2 summarizes the phenotype observed in the index cases and the affected carriers.

In the IC group $(n=65)$, the mean age at onset was 30.0 years $( \pm 16.5)$. Thirty-four patients $(52.3 \%)$ had developed symptoms before 30 years of age. The mean age at diagnosis was 44.2 years $( \pm 15.9)$. The mean disease duration at inclusion was 7.2 years $( \pm 6.9)$. The mean diagnosis delay was 14.2 years (ranging 1-49 years). The type of first symptoms experienced by patients was scapulo-humeral weakness in 40 patients (61.5\%), facial weakness in 3 patients (4.6\%), lower limb weakness in 18 patients (27.7\%), muscle pain in 2 patients (3.0\%), clumsiness in one patient $(1.5 \%)$ and not informed in one patient. When comparing the, ICs carrying 6-8 RUs $(n=49)$ and those carrying 9-10 RUs $(\mathrm{n}=16)$, we found a higher proportion of patients developing symptoms before 30 years in the 6-8 RUs subgroup (59.2\%) than in the 9-10 RUs subgroup (31.3\%), although it did not reach statistical significance $(\mathrm{p}=0.052)$. Of 7 patients in the IC group $(2$ men and 5 women) who were wheelchair-bound (mean age 60.9 years), six of them belonged to the 6-8 RUs subgroup.

The relatives were divided into the two subgroups by number of RUs: 6-8 RUs $(n=40)$ and 9-10 RUs $(n=19)$.

\section{Study of penetrance}

Overall, among the 124 carriers, 99 subjects were clinically affected (65 ICs and 34 relatives), which leads to estimate the penetrance of the disease in the whole group 


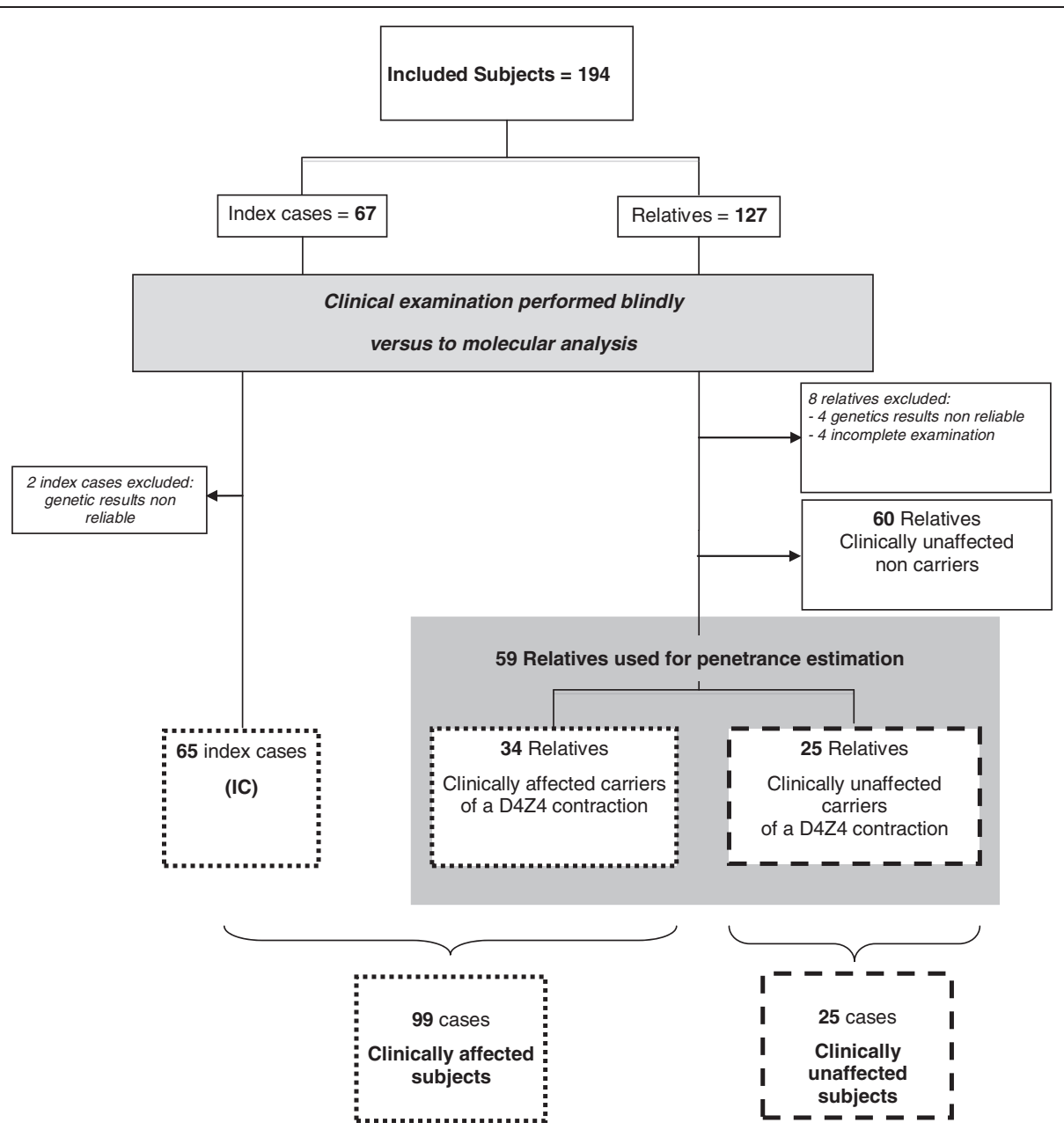

Figure 1 Repartition of subjects included in the study.

of 6-10 RUs at 57\% (if ICs are excluded) or 79\% (if ICs are included). In the 6-8 RUs subgroup, penetrance varied from 62 to $83 \%$ whereas in the 9-10 RUs subgroup, it ranged from $47 \%$ to $71 \%$. We found a higher proportion of clinically unaffected versus clinically affected carriers in the 9-10 RUs group than in the 6-8 RUs group, although it did not reach the statistical significance.

An age-related cumulative frequency of penetrance was estimated using the Kaplan-Meier method on the data obtained from $65 \mathrm{ICs}$ and 25 unaffected carriers
(Figure 2 and Table 3). In the 6-8 RUs subgroup, the risk of developing motor impairment was $35 \%$ by age 20 , $60 \%$ by age 40 and $81 \%$ by age 60 , whereas in the $9-10$ RUs subgroup, the risk was $19 \%$ by age $20,44 \%$ by age 40 , and $71 \%$ by age 60 .

\section{Influence of age, sex, environmental factors and degree of kinship on penetrance}

In order to evaluate the influence of age, sex, and environmental factors on penetrance, we compared the affected

Table 1 Descriptive statistics of age and sex

\begin{tabular}{|c|c|c|c|c|c|}
\hline & ICs n (\%) & Affected carriers n (\%) & Unaffected carriers n (\%) & Non carriers n (\%) & $P$ value \\
\hline & 65 & 34 & 25 & 60 & \\
\hline Gender & & & & & 0.002 \\
\hline Male & $44(68 \%)^{a, b}$ & $18(53 \%)$ & $7(28 \%)^{a}$ & $24(40, \%)^{b}$ & \\
\hline Female & $21(32 \%)$ & $16(47 \%)$ & $18(72 \%)$ & $36(60 \%)$ & \\
\hline Age, years (mean \pm standard deviation) & $51.2 \pm 14.9$ & $50.5 \pm 17.4$ & $47.6 \pm 19.5$ & $46.9 \pm 17.4$ & 0.482 \\
\hline
\end{tabular}

Overall age comparison: Kruskall-Wallis test $p=0.482$. Overall gender comparison: chi square test $p=0.002$. Significant pairwise comparisons: ${ }^{a}$ ICs versus unaffected carriers $p=0.0007$ and ${ }^{b}$ ICs versus non carriers $p=0.001$. 
Table 2 Phenotype in index cases and affected carriers at the time of examination

\begin{tabular}{lll}
\hline & $\begin{array}{l}\text { ICs } \\
\text { n (\%) }\end{array}$ & $\begin{array}{l}\text { Affected carriers } \\
\text { n (\%) }\end{array}$ \\
\hline Facial weakness & $49(75 \%)$ & $20(59 \%)$ \\
Scapular weakness & $65(100 \%)$ & $32(94 \%)$ \\
Pectoral weakness & $55(85 \%)$ & $20(59 \%)$ \\
Abdominal weakness & $51(78.5 \%)$ & $20(59 \%)$ \\
Asymmetry & $49(75 \%)$ & $23(68 \%)$ \\
Steppage & $31(48 \%)$ & $2(6 \%)$ \\
\hline
\end{tabular}

and the unaffected carriers. We found no difference with the age criterion. Seventy two percent of the unaffected carriers (18/25) were women, although it did not reach statistical significance $(p=0.055)$. There was no difference of smoking habits or intake of alcohol between the two groups. Regarding medical history (high blood pressure, cardiovascular diseases, diabetes mellitus, cancer, thyroid disorders, trauma, and anesthesia), level of physical activity, and exposure to medication (analgesics, anti-inflammatory, cholesterol-lowering drugs, or anti-depressants), there was no difference either. We did not find any effect of the degree of kinship.

The risk of developing FSHD between clinically affected patients (ICs and affected carriers, $\mathrm{n}=99$ ) and clinically unaffected carriers $(n=25)$ was evaluated by logistic regression analysis. There was no significant effect of age on the risk of developing motor impairment $(\mathrm{OR}=1.02$, $\mathrm{CI}=0.99-1.05)$. The risk of developing symptoms of FSHD was 4.6-fold higher $(\mathrm{p}=0.0041$; OR $=4.6, \mathrm{CI}=1.6$ 12.8) for men than for women, suggesting a significant effect of sex in the disease. Regarding the influence of the number of RUs on penetrance, the risk of developing symptoms of FSHD was not significantly increased in patients with 6-8 RUs versus in patients with 9-10 RUs $(\mathrm{p}=0.72 ; \mathrm{OR}=2.01, \mathrm{CI}=0.74-5.50)$.

\section{Discussion}

We have conducted a cross-sectional multicenter study of the penetrance in FSHD1 patients carrying contracted alleles of 6-10 RUs. In this range, the penetrance was estimated to be $57 \%$ (if ICs are excluded) or $79 \%$ (if ICs are included). The penetrance was lower in patients with 9-10 RUs (47\% if ICs are excluded or 71\% if ICs are included) than in those with 6-8 RUs (62\% if ICs are excluded or $83 \%$ if ICs are included).

In the premolecular era, the penetrance of FSHD1 was considered to be age-related and almost complete at age 20 [17]. When including D4Z4 analyses, the penetrance was shown to be incomplete but higher than $80 \%$ $[13,14,18,19]$. More recently, the penetrance was shown to be lower than expected. In a retrospective study of 85 Japanese families, including sporadic and familial cases, Goto et al. [20] observed a penetrance of 59\%. Of late, a Greek survey of 133 patients estimated that the penetrance ranged from 50 (ICs excluded) to $77 \%$ (ICs included) in FSHD1 patients carrying a contracted allele $\leq 38 \mathrm{~kb}$ [9]. In addition, in a large Italian cohort, Ricci et al. estimated the penetrance in patients with alleles of 1-8 RUs [8]. The estimated risk of developing motor impairment by age 60 for relatives carrying 1-3 RUs and 4-8 RUs was $96.2 \%$ and $71.5 \%$, respectively. These results suggest an inverse relationship between penetrance and size of the contracted allele.

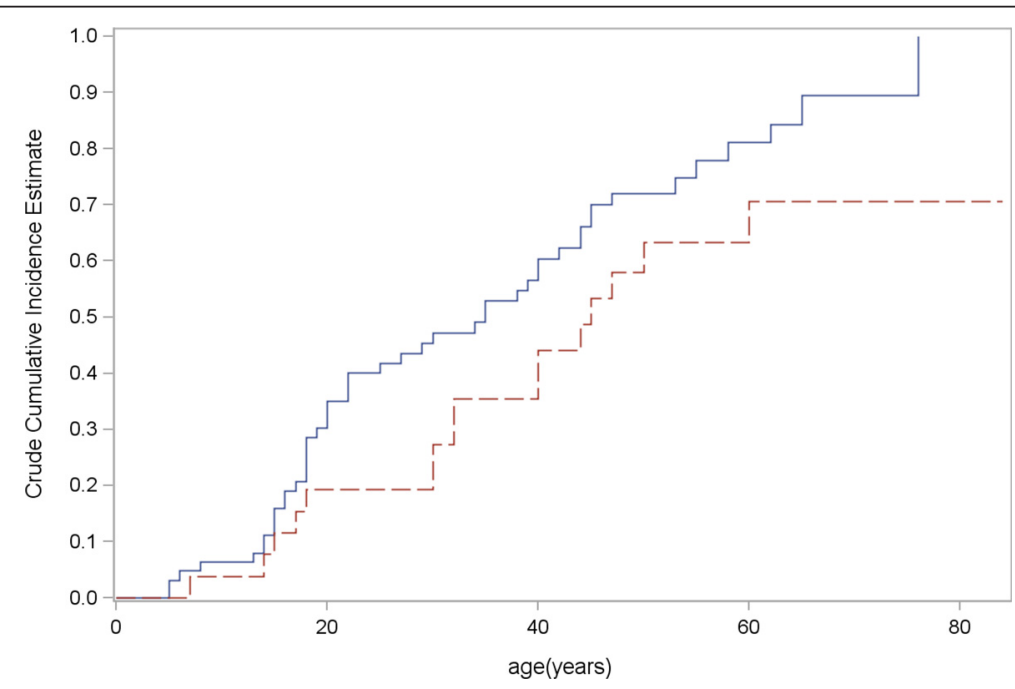

Figure 2 Age-related cumulative risk of being clinically affected according to the number of RUs. Estimates obtained on $65 \mathrm{IC}$ and 25 unaffected carriers using the Kaplan-Meier analysis. Blue line refers to 6-8 RUs; Red line refers to 9-10 RUs. Carriers 6-8 RUs versus 9-10 RUs, Log-Rank test $P=0.0584$. 
Table 3 Estimates of the age-specific cumulative risk using Kaplan-Meier analysis

\begin{tabular}{|c|c|c|c|c|}
\hline \multirow{2}{*}{$\begin{array}{l}\text { Age } \\
\text { (years) }\end{array}$} & \multicolumn{2}{|c|}{ 6-8 RUs } & \multicolumn{2}{|c|}{ 9-10 RUs } \\
\hline & Risk & $95 \% \mathrm{Cl}$ & Risk & $95 \% \mathrm{Cl}$ \\
\hline 20 & 35.1 & $(25.0 ; 49.1)$ & 19.2 & $(8.7 ; 42.3)$ \\
\hline 30 & 47.2 & $(36.1 ; 61.6)$ & 27.3 & $(14.5 ; 51.4)$ \\
\hline 40 & 60.4 & $(48.9 ; 74.5)$ & 44.0 & $(28.2 ; 68.7)$ \\
\hline 50 & 72.0 & $(61.1 ; 85.0)$ & 63.3 & $(46.1 ; 86.9)$ \\
\hline 60 & 81.1 & $(70.2 ; 93.7)$ & 70.6 & $(52.7 ; 94.5)$ \\
\hline 70 & 89.5 & $(78.9 ; 100)$ & & \\
\hline
\end{tabular}

In the range of the largest pathological alleles, penetrance data are scarce to date. Clinical reports have suggested that the penetrance in this range is low, but it has never been calculated through large family studies $[10,11,21,22]$. Our cohort is the largest one including FSHD1 families with contracted alleles in the upper range of pathological alleles (9-10 RUs). We showed that the age-related cumulative risk of being clinically affected was lower in patients with 9-10 RUs than in patients with 6-8 RUs (70\% versus $81 \%$ at age 60 ), although the difference was not statistically significant. To compare, in the Italian survey, the risk of being clinically affected in patients with 7-8 RUs was lower than in patients with 4-6 RUs (63\% versus $71 \%$ at age 60 ) [8]. Our study suggests the same inverse relationship between penetrance and size of the contracted allele in the range 9-10 RUs, as observed for alleles smaller than 8 RUs.

In our study, the mean age of onset (30 yo) was higher than reported in previous studies, ie 16 years [23] and 23 years [24]. In our cohort, only $52 \%$ of ICs had developed symptoms before 30 , contrasting with the $70 \%$ reported elsewhere [25]. Several studies in FSHD1 have shown a significant correlation between age at onset and EcoRI fragment size [26]. All the previous studies included patients with alleles $<6$ RUs while we excluded them from our cohort. Accordingly, our data suggest that the disease onset in patients with the largest alleles is later than in those with the shortest alleles. In agreement with previous studies, we found a significant effect of sex on FSHD1 penetrance, with women being less clinically affected than men $[2,8,14,18,19]$. Our data confirm the influence of sex, even in alleles in the upper range.

The concordance between the clinical status determined by the neurological examination and the results of the genetic analysis was complete in this study, emphasizing the high predictive value of clinical expertise in accurate FSHD diagnosis. Regarding the mode of onset of the disease, the first symptom was scapulohumeral weakness in $61 \%$ of patients, as in previous studies [8,9]. The onset in the lower limbs was higher in our series
(28\%) than previously reported (11.7 to $16.7 \%)$ [8,9]. Furthermore, only $10.7 \%$ of our ICs were wheelchair-bound (mean age of 50 years; mean disease duration of 7 years) while previous studies reported that approximately $20 \%$ of FSHD1 patients become wheelchair-bound by the age of $50[9,27,28]$. We hypothesize that in the range of the upper alleles, the clinical course of the disease is less severe in line with the fact that the most severe phenotypes usually result from the shortest alleles (1-3 RUs) [29,30].

In addition, we searched for an environmental effect that could influence the FSHD1 penetrance. We did not find any effects of drugs, anesthesia, surgery or traumatisms on the penetrance. Nevertheless, the number of patients included in this study was too small for epidemiological studies. Further investigations in large cohorts are needed to exclude the effect of environmental factors on FSHD. Besides, previous studies suggested that epigenetic mechanisms or modifier genes influence the clinical expression of FSHD [31].

Recently, a novel gene called SMCHD1 has been identified as causative in FSHD without D4Z4 contraction (FSHD2), and has also been proposed as a modifier in FSHD1 [32,33]. In our study, penetrance could have been influenced by epigenetic factors, which should be further explored.

\section{Conclusion}

In conclusion, penetrance is low in patients with alleles in the upper range and lower in women than men. In this range, the age of onset is later and the course of the disease is less severe. Knowledge of penetrance and expressivity of FSHD1 is of crucial importance for genetic counseling and clinical management of patients and families. Our findings indicate that great caution must be taken concerning predictive and prognostic information provided to FSHD1 carriers of the largest alleles. Deeper investigations of genotype-phenotype correlations are ongoing in our cohort in order to provide a precise characterization of this patient subgroup.

\section{Abbreviations}

FSHD: Facioscapulohumeral muscular dystrophy; FSHD1: Facioscapulohumeral muscular dystrophy type 1; IC: Index case; RUs: Repeat units.

\section{Competing interests}

All the authors declare they have no competing interests.

\section{Authors' contributions}

ESC included patients, performed neurological examination, analyzed the data and wrote the paper. KN contributed to design of the project,

performed genetic consulting and analysis, analyzed the data and revised the manuscript. RB contributed to the design of the project, performed genetic analysis, and analyzed the data and revised the manuscript. EJ contributed to the design of the project, conducted and performed the statistical analysis and revised the manuscript. GS included patients, performed neurological examination and revised the manuscript. ANP included patients, performed neurological examination and analyzed the data. JN included patients, performed neurological examination and analyzed the data. EC contributed to the statistical analysis. EO included 
patients, performed neurological examination and analyzed the data. FB included patients, performed neurological examination and analyzed the data. SS included patients, performed neurological examination and analyzed the data. AEL included patients, performed neurological examination and analyzed the data. CD included patients, performed neurological examination and analyzed the data. CT included patients, performed neurological examination and analyzed the data. CV included patients, performed neurological examination and analyzed the data. FM contributed to the genetic analysis and revised the manuscript. MB contributed to the genetic analysis and revised the manuscript. MCAB included patients, performed neurological examination and analyzed the data. XF included patients, performed neurological examination and analyzed the data. TK included patients, performed neurological examination and analyzed the data. NL contributed to the design of the project and revised manuscript. JP contributed to the design of the project and revised manuscript. SA was principal investigator, designed and coordinated the project, included patients, performed neurological examination, analyzed the data and wrote the paper. All authors read and approved the final manuscript.

\section{Acknowledgement}

This work was supported by a grant from the AFM-Téléthon (Association Française contre les Myopathies) ( $\left.N^{\circ} 10906\right)$. For her hard work and engagement in this study, we are grateful to Dr Isabelle Pénisson-Besnier who passed away. We would like to thank C. Vovan, C. M'Chinda (Department of Medical Genetics, Timone University Hospital, Marseille) and C. Castro (Aix Marseille University, INSERM, GMGF UMR_S 910, Marseille) for their significant contribution in molecular analysis. The authors warmly thank all patients and their families for participation

\section{Author details}

${ }^{1} \mathrm{AP}-\mathrm{HM}$, Reference Center of Neuromuscular Disorders and ALS, Timone University Hospital, Aix-Marseille University, 264 rue Saint-Pierre, Marseille, Cedex 05 13385, France. ${ }^{2}$ Aix Marseille Université - Inserm UMR_S 910 Medical Genetics and Functional Genomics, Marseille, France. ${ }^{3}$ AP-HM, Department of Medical Genetics, Timone University Hospital, Marseille, France. ${ }^{4} \mathrm{CIC}-$ UPCET, Timone University Hospital, AP-HM, UMR CNRS Aix-Marseille University 6193, Marseille, France. ${ }^{5}$ Reference Center of Neuromuscular Disorders, CHU of Bordeaux, Pessac, France. ${ }^{6}$ Centre de Référence des Maladies Neuromusculaires Nantes-Angers, Service de Neurologie, CHU d'Angers, Angers, France. ${ }^{7}$ Nerve-Muscle Unit, Department of Clinical Neurosciences, Lausanne University Hospital (CHUV), Lausanne, Switzerland. ${ }^{8}$ Croix-Rousse Hospital, Lyon, France. ${ }^{9}$ Electroneuromyography and Neuromuscular Department, GHE Neurologic hospital, Lyon, Bron Cedex 69677, France. ${ }^{10}$ Neuromuscular Disease Specialized Center, Nice University Hospital, Nice, France. ${ }^{11}$ Reference Center of Neuromuscular Disorders, Neurology Department, Hautepierre Hospital, Strasbourg, France. ${ }^{12}$ Reference Center of Neuromuscular Disorders, $\mathrm{CHU}$ of Toulouse, Toulouse, France.

\section{Received: 14 October 2014 Accepted: 29 December 2014} Published online: 21 January 2015

\section{References}

1. Mostacciuolo ML, Pastorello E, Vazza G, Miorin M, Angelini C, Tomelleri G, et al. Facioscapulohumeral muscular dystrophy: epidemiological and molecular study in a north-east Italian population sample. Clin Genet. 2009;75:550-5.

2. Padberg GW. Facioscapulohumeral Disease. Leiden, The Netherlands: University of Leiden; 1982.

3. van Deutekom JC, Wijmenga C, van Tienhoven EA, Gruter AM, Hewitt JE, Padberg GW, et al. FSHD associated DNA rearrangements are due to deletions of integral copies of a $3.2 \mathrm{~kb}$ tandemly repeated unit. Hum Mol Genet. 1993;2:2037-42.

4. Lemmers RJ, de Kievit P, Sandkuijl L, Padberg GW, van Ommen GJ, Frants RR, et al. Facioscapulohumeral muscular dystrophy is uniquely associated with one of the two variants of the $4 \mathrm{q}$ subtelomere. Nat Genet. 2002;32:235-6.

5. Lemmers RJ, Wohlgemuth M, Frants RR, Padberg GW, Morava E, van der Maarel SM. Contractions of D4Z4 on 4qB subtelomeres do not cause facioscapulohumeral muscular dystrophy. Am J Hum Genet. 2004;75:1124-30.

6. Lemmers RJ, van der Vliet PJ, Klooster R, Sacconi S, Camaño P, Dauwerse JG, et al. A unifying genetic model for facioscapulohumeral muscular dystrophy. Science. 2010;329:1650-3.
7. Scionti I, Greco F, Ricci G, Govi M, Arashiro P, Vercelli L, et al. Large-scale population analysis challenges the current criteria for the molecular diagnosis of fascioscapulohumeral muscular dystrophy. Am J Hum Genet. 2012;90:628-35

8. Ricci G, Scionti I, Sera F, Govi M, D'Amico R, Frambolli I, et al. Large scale genotype-phenotype analyses indicate that novel prognostic tools are required for families with facioscapulohumeral muscular dystrophy. Brain. 2013;136:3408-17.

9. Sakellariou P, Kekou K, Fryssira H, Sofocleous C, Manta P, Panousopoulou A, et al. Mutation spectrum and phenotypic manifestation in FSHD Greek patients. Neuromuscul Disord. 2012;22:339-49.

10. Butz M, Koch MC, Muller-Felber W, Lemmers RJ, van der Maarel SM, Schreiber H. Facioscapulohumeral muscular dystrophy. Phenotype-genotype correlation in patients with borderline D4Z4 repeat numbers. J Neurol. 2003;250:932-7.

11. Vielhaber S, Jakubiczka S, Schroder JM, Sailer M, Feistner H, Heinze HJ, et al. Facioscapulohumeral muscular dystrophy with EcoRI/BInl fragment size of more than 32 kb. Muscle Nerve. 2002;25:540-8.

12. Felice $\mathrm{KJ}$, Whitaker $\mathrm{CH}$. The clinical features of facioscapulohumeral muscular dystrophy associated with borderline (>/=35 kb) 4q35 EcoRl fragments. J Clin Neuromuscul Dis. 2005;6:119-26.

13. Galluzzi G, Deidda G, Cacurri S, Colantoni L, Piazzo N, Vigneti E, et al. Molecular analysis of $4 q 35$ rearrangements in fascioscapulohumeral muscular dystrophy (FSHD): application to family studies for a correct genetic advice and a reliable prenatal diagnosis of the disease. Neuromuscul Disord. 1999;9:190-8.

14. Ricci E, Galluzzi G, Deidda G, Cacurri S, Colantoni L, Merico B, et al. Progress in the molecular diagnosis of facioscapulohumeral muscular dystrophy and correlation between the number of Kpnl repeats at the $4 \mathrm{q} 35$ locus and clinical phenotype. Ann Neurol. 1999;45:751-7.

15. Padberg GW, Lunt PW, Koch M, Fardeau M. Diagnostic criteria for facioscapulohumeral muscular dystrophy. Neuromuscul Disord. 1991;1:231-4.

16. Scionti I, Fabbri G, Fiorillo C, Ricci G, Greco F, D'Amico R, et al. Facioscapulohumeral muscular dystrophy: new insights from compound heterozygotes and implication for prenatal genetic counselling. J Med Genet. 2012;49:171-8

17. Lunt PW, Compston DA, Harper PS. Estimation of age dependent penetrance in facioscapulohumeral muscular dystrophy by minimising ascertainment bias. J Med Genet. 1989;26:755-60.

18. Tonini MM, Passos-Bueno MR, Cerqueira A, Matioli SR, Pavanello R, Zatz M. Asymptomatic carriers and gender differences in facioscapulohumeral muscular dystrophy (FSHD). Neuromuscul Disord. 2004;14:33-8.

19. Zatz M, Marie S, Cerqueira A, Vainzof M, Pavanello R, Passos BM. The facioscapulohumeral muscular dystrophy (FSHD1) gene affects males more severely and more frequently than females. Am J Med Genet. 1998;77:155-61.

20. Goto K, Nishino I, Hayashi YK. Very low penetrance in 85 Japanese families with facioscapulohumeral muscular dystrophy 1A. J Med Genet. 2004;41:e12.

21. Busse K, Kohler J, Stegmann K, Pongratz D, Koch MC, Schreiber H. An inherited 4q35-EcoRI-DNA-fragment of $35 \mathrm{~kb}$ in a family with a sporadic case of facioscapulohumeral muscular dystrophy (FSHD). Neuromuscul Disord. 2000;10:178-81

22. Vitelli F, Villanova M, Malandrini A, Bruttini M, Piccini M, Merlini L, et al. Inheritance of a 38-kb fragment in apparently sporadic facioscapulohumeral muscular dystrophy. Muscle Nerve. 1999;22:1437-41.

23. Padberg GW, Frants RR, Brouwer OF, Wijmenga C, Bakker E, Sandkuijl LA. Facioscapulohumeral muscular dystrophy in the Dutch population. Muscle Nerve. 1995;2(Suppl):81-4.

24. Pastorello E, Cao M, Trevisan CP. Atypical onset in a series of 122 cases with FacioScapuloHumeral Muscular Dystrophy. Clin Neurol Neurosurg. 2012;114:230-4.

25. The FSH-DY group. A prospective, quantitative study of the natural history of facioscapulohumeral muscular dystrophy (FSHD): implications for therapeutic trials. Neurology. 1997;48:38-46.

26. Lunt PW, Jardine PE, Koch MC, Maynard J, Osborn M, Williams M, et al. Correlation between fragment size at D4F104S1 and age at onset or at wheelchair use, with a possible generational effect, accounts for much phenotypic variation in 4q35-facioscapulohumeral muscular dystrophy (FSHD). Hum Mol Genet. 1995;4:951-8.

27. Lunt PW, Harper PS. Genetic counselling in facioscapulohumeral muscular dystrophy. J Med Genet. 1991;28:655-64. 
28. Statland JM, Tawil R. Risk of functional impairment in Facioscapulohumeral muscular dystrophy. Muscle Nerve. 2014;49:520-7.

29. Klinge L, Eagle M, Haggerty ID, Roberts CE, Straub V, Bushby KM. Severe phenotype in infantile facioscapulohumeral muscular dystrophy. Neuromuscul Disord. 2006;16:553-8

30. Trevisan CP, Pastorello E, Tomelleri G, Vercelli L, Bruno C, Scapolan S, et al. Facioscapulohumeral muscular dystrophy: hearing loss and other atypical features of patients with large 4q35 deletions. Eur J Neurol. 2008:15:1353-8.

31. van der Maarel SM, Tawil R, Tapscott SJ. Facioscapulohumeral muscular dystrophy and DUX4: breaking the silence. Trends Mol Med. 2011;17:252-8.

32. Lemmers RJ, Tawil R, Petek LM, Balog J, Block GJ, Santen GW, et al. Digenic inheritance of an SMCHD1 mutation and an FSHD-permissive D4Z4 allele causes facioscapulohumeral muscular dystrophy type 2. Nat Genet. 2012:44:1370-4.

33. Sacconi S, Lemmers RJ, Balog J, van der Vliet PJ, Lahaut P, van Nieuwenhuizen MP, et al. The FSHD2 gene SMCHD1 is a modifier of disease severity in families affected by FSHD1. Am J Hum Genet. 2013;93:744-51.

\section{Submit your next manuscript to BioMed Central and take full advantage of:}

- Convenient online submission

- Thorough peer review

- No space constraints or color figure charges

- Immediate publication on acceptance

- Inclusion in PubMed, CAS, Scopus and Google Scholar

- Research which is freely available for redistribution 